\title{
Gaslift pump making use of phase change of working fluid
}

\author{
Shigeo Hosokawa*, Shudai Fujimoto, Akio Tomiyama \\ Department of Mechanical Engineering, Kobe University, 1-1 Rokkodai, Nada, Kobe, Hyogo 657-8501, Japan \\ *Corresponding author. Tel.: +81 78803 6132; \\ fax: +81 78803 6132; email: hosokawa@mech.kobe-u.ac.jp
}

\begin{abstract}
Improvement of efficiency of a gaslift pump is required for lifting submarine resources economically. We propose a gaslift pump utilizing phase change of working fluid to improve the efficiency by avoiding air compression. A working fluid, which has a boiling point lower than surrounding temperature, is supplied as liquid to an evaporator near the bottom of a riser pipe through an adiabatic pipe. The working fluid boils at the evaporator due to heating from the surrounding liquid. The generated gas is injected into the riser pipe instead of compressed air and forms gas-liquid two-phase flow, by which the water is drawn from the bottom opening of the riser pipe. The working fluid is separated from the drawn water at the top of the pipe, and it flows into a cooler to supply the condensed liquid into the adiabatic pipe again. This pump needs the energy only to cool the working fluid, i.e. no energy is required for compressing gas. The feasibility of the pump is confirmed through the experiments using a prototype gaslift pump using a hydrofluorocarbon as the working fluid. Prediction of the flow rate of drawn water is also carried out.
\end{abstract}

Keywords: Gaslift pump, Phase change, Two-phase flow, Slug flow, Submarine resource 


\section{Introduction}

Submarine resources such as manganese nodules, hydrothermal deposits, CRC (cobalt-rich crust), methane hydrate and REE (rare-earth elements) rich deep-sea mud are paid attention to as the next generation resources. However, reduction in the mining costs is indispensable for increasing the profitability (Rona, 2003). An airlift pump is one of the promising pump systems for mining the submarine resources (Saito, 1986). The pump draws liquid by buoyancy force acting on gas injected into a riser pipe, and it has several advantages such as simple structure, no moving parts, no structure in the flow channel and low maintenance cost. Hence the pumps can be utilized not only for mining submarine resources but also for dredging a harbor or a dam lake, pumping water from a well and lifting corrosive substances in chemical industries. On the other hand, the number of practical systems is small due to its low efficiency (Kassab et al., 2007). Many researches have been carried out to understand performances of airlift pumps (Todoroki, et al., 1972; Hatakeyama \& Masuyama, 1989; Kassab, et al., 2009) and the fundamental characteristics has been clarified through these studies. Numerical methods for prediction of gas-liquid-solid three phase flow in the main pipe of airlift pump have been developed by several researches such as Tomiyama et al. (1991) and Hatakeyama et al. (1999). Although several researchers have attempted to improve its efficiency (Cho et al., 2009, Sadatomi et al., 2008, 2013), large work for injecting compressed air into the main pipe makes it difficult to improve the efficiency drastically. Since the air-injection pressure increases with the depth of water, the efficiency becomes very low when applying it to deep sea area.

In this study, we propose a gaslift pump which uses phase change of working fluid. A small scale gaslift pump using a hydro-fluorocarbon as the working fluid is developed to examine the feasibility of the proposed system. The flow rates of the drawn water are measured at various flow rates of the working fluid and using three pipes with different diameters. Experiments using compressed air, i.e., a standard airlift pump, are also carried out for comparison. The numerical 
method proposed by Hatakeyama and Masuyama (1989) is applied to the present system to predict the performance of the gaslift pump.

\section{Gaslift pump utilizing phase change of working fluid}

We propose a gaslift pump making use of phase change of working fluid, the concept of which is shown in Fig. 1. A fluid, which has a boiling point $T_{B}$ lower than the temperature $T_{0}$ of surrounding liquid, is used as the working fluid instead of compressed air. The fluid is supplied as liquid to the evaporator near the gas injection point of the main pipe (riser pipe) through the adiabatic pipe in which the liquid flows downward. When the density $\rho_{L}$ of the liquid is higher than that of the liquid, $\rho$, around the gaslift pump, the liquid falls automatically in the adiabatic pipe due to the gravity. The working fluid boils in the evaporator by heat input from the surrounding liquid. Since the elevation of the exit of the evaporator is higher than that of the inlet, the generated gas flows into the main pipe through the exit. The gas injected into the main pipe forms gas-liquid twophase flow and draws the surrounding liquid from the bottom opening of the main pipe. The drawn liquid is pumped up to the top of the main pipe and the gas (working fluid) is separated from the liquid at the phase separation section. The separated gas flows into the condenser and is condensed by heat removal. The condensed liquid flows into the adiabatic pipe again.

This gaslift pump only needs the energy to condense the working fluid, unlike standard airlift pumps which require the energy for compressing the air. Requirements for the working fluid are as follows: (1) $\rho_{L}$ is higher than $\rho_{0}$, (2) $T_{B}$ is lower than $T_{0}$ at the depth of the evaporator and (3) it is insoluble or slightly soluble in the surrounding liquid. Low latent heat of the working fluid is desirable to increase the efficiency. No impact of the working fluid on the environment is also required for application to mining submarine resources or dredging a harbor or a lake. 


\section{Experimental apparatus}

Feasibility experiments were carried out by using a hydro-fluorocarbon (HFC-245fa, the gas density $\rho_{G}=5.92 \mathrm{~kg} / \mathrm{m}^{3}, \rho_{L}=1365 \mathrm{~kg} / \mathrm{m}^{3}, T_{B}=288 \mathrm{~K}$ at atmospheric pressure, latent heat: 197 $\mathrm{kJ} / \mathrm{kg}$, specific heat (gas): $0.878 \mathrm{~kJ} / \mathrm{kg} / \mathrm{K}$, specific heat (liquid): $1.36 \mathrm{~kJ} / \mathrm{kg} / \mathrm{K}$ ) as the working fluid. A schematic of the experimental apparatus is shown in Fig. 2. The prototype gaslift pump was installed in the large water tank of $0.2 \times 0.2 \times 1.0 \mathrm{~m}^{3}$. The volume of the tank was much larger than the volume of drawn water, so that the change in water level was negligible during the experiments. The experiments were carried out for the main pipes with the inner diameters, $D=8,11$ and 16 mm, while the length of the pipes was fixed at $1020 \mathrm{~mm}$ (the length $H_{d}$ between the water surface and the pipe exit: $300 \mathrm{~mm}$, the length $L^{\prime}$ ' between the bottom and the gas injection point: $20 \mathrm{~mm}$ ). The four Peltier devices (HB Co., TEC1-12708) with heat sinks were installed in the condenser to cool the hydro-fluorocarbon gas. The vacuum insulation tube was used for the adiabatic pipe and its inner diameter was $9 \mathrm{~mm}$. The evaporator consisted of a polycarbonate body and two aluminum plates (thickness: $0.5 \mathrm{~mm}$ ) mounted on the two sides of the body. The gas flow rate was controlled by changing the liquid level in the evaporator, i.e., heat-transfer area for the liquid. The liquid flow rate of the working fluid was controlled so as to balance with the gas flow rate by adjusting the valve just below the condenser. The separator consisted of the closed and open tanks, and the main pipe was connected to the closed tank. The drawn water was separated in the closed tank and flowed into the large water tank through the open tank. The water level in the closed tank was adjusted to that in the open tank by using the valve at the exit of the closed tank. Hence, the pressure in the closed tank was fixed at the atmospheric pressure. The reserve bag was connected to the condenser to mitigate flow rate oscillation. Experiments for a standard airlift pump were also carried out by replacing the condenser, adiabatic pipe and evaporator to a compressor and an air supply pipe. 
The wet gas flowmeter (Shinagawa, WS-1A-Da) was installed between the separator and the condenser to measure the gas flow rate of the working fluid. Water flow rate was calculated from its volume and time measured by using a graduated cylinder and a stopwatch at the exit of the separator. Uncertainties at the 95\% confidence level in the measurements of gas and water flow rates were $0.2 \%$ and $0.5 \%$, respectively. The water (surrounding liquid) temperature $T_{0}$ ranged from 298 to $313 \mathrm{~K}$ to examine the effect of $T_{0}$ on the flow rate of drawn water. The temperatures $T_{1}$, $T_{2}, T_{3}$ and $T_{4}$ at the inlet of the adiabatic pipe, the bottom of the adiabatic pipe, the exit of the evaporator and the top of the separator, respectively, were monitored and recorded by a data logger (Graphtec, midi Logger GL900) during the experiments. T type sheath thermocouples (class 1, \pm 0.5 ${ }^{\circ} \mathrm{C}$ ) were used for the temperature measurements.

\section{Results and discussion}

Photographs of flows in the evaporator, the main pipe and the separator (the exit of the main pipe) are shown in Fig. 2. The pipe diameter $D$, the temperature $T_{0}$ of surrounding water and the volume flow rate $Q_{G}$ of gas (working fluid) were $11 \mathrm{~mm}, 303 \mathrm{~K}$ and $2.0 \times 10^{-5} \mathrm{~m}^{3} / \mathrm{s}$, respectively. Gas bubbles were generated at the side plates in the evaporator and the generated gas was supplied to the main pipe (the left bottom photograph). The slug flow was formed in the main pipe (the middle photograph), and the water was pumped up (the left top photograph) to the separator by the gas. The volume flow rate $Q_{L}$ of drawn water were $2.1 \times 10^{-5} \mathrm{~m}^{3} / \mathrm{s}$. The electric power supplied to the Peltier devices was $29 \mathrm{~W}$. Figure 3 shows time variations of temperatures and $Q_{G}$. The dashed line indicates the boiling temperature at the pressure in the evaporator. The temperature and the flow rate were kept almost constant values and the fluctuations were small. Hence, the flow induced by the present gaslift pomp was stable, whereas there was intermittency due to slug flow in a short

time scale. These results clearly indicate the feasibility of the present gaslift pump making use of phase change of working fluid. 
The temperature gradually increased as the working fluid flows from the inlet of the adiabatic pipe $\left(T_{1}\right)$ to the exit of the main pipe $\left(T_{4}\right)$. The temperature difference between the inlet and outlet of the condenser was almost the same as $T_{4}-T_{1}$ and was about $14 \mathrm{~K}$ for the condition shown in Fig. 3. This temperature difference balanced with the heat removed in the condenser, and its decrease improved the efficiency of the system. Possible ways to improve the efficiency are (1) to use a working fluid which has a boiling temperature close to the surrounding fluid and (2) to employ an adiabatic pipe for the main pipe by which the temperature rise in the main pipe is minimized (the heat transfer between the drawn liquid and gas increases the gas temperature and the adiabatic expansion of the gas phase decreases the temperature).

Visualized images of flow in the main pipe are shown in Fig. 4. The flow pattern of the twophase flow in the main pipe was slug flow for all the conditions except the highest $Q_{G}$ condition for $D=8 \mathrm{~mm}$ in which the flow pattern was the transition from slug flow to churn flow. The lengths of large bubbles and the number of small bubbles tended to increase with $Q_{G}$. Fluctuation of the interface of large bubbles was observed in high $Q_{L}$ conditions.

The relations between the flow rates, $Q_{G}$ and $Q_{L}$, of the working fluid and the drawn water are shown in Fig. 5 together with those of the standard airlift pump using compressed air. The $Q_{L}$ increased with $Q_{G}$ in all the conditions, and the gradient of $Q_{L}$ became small as $Q_{G}$ increased. The $Q_{L}$ in the range of $Q_{G}>4 \mathrm{~m} / \mathrm{s}$ for $D=8 \mathrm{~mm}$ little depended on $Q_{G}$, where Taylor bubbles longer than $10 D$ were frequently formed as shown in Fig. 4. Further increase in $Q_{G}$ might decrease $Q_{L}$ (ex. Todoroki et al. (1972)). No dependence on the surrounding liquid temperature $T_{0}$ was observed due to the small change in fluid properties in the present experimental range, while the increase in $T_{0}$ increased the maximum $Q_{G}$ due to an increased heat transfer rate at the evaporator. The $Q_{L}$ increased with $D$ at a constant $Q_{G}$ due to decrease in the frictional loss for $Q_{G}>3.5 \times 10^{-5} \mathrm{~m}^{3} / \mathrm{s}$, whereas it decreased with increasing $D$ in the low $Q_{G}$ conditions. The $Q_{L}$ of the present gaslift pump 
was almost the same as that of the standard airlift pump for all the pipe diameters. This indicates that the knowledge on airlift pumps can be applicable to the present gaslift pump.

The flow rate of drawn water was predicted by using a one-dimensional numerical method based on a drift flux model proposed by Hatakeyama et al. (1989). The simulated domain and parameters are summarized in Fig. 6. The method was based on the one-dimensional mass conservations of two-phase mixture and gas phase and the momentum conservation of two-phase mixture. The mixture mass conservation is given by

$$
\frac{\partial \rho_{m}}{\partial t}+\frac{\partial \rho_{m} V_{m}}{\partial z}=0
$$

where $t$ is the time, $z$ the elevation from the bottom of the main pipe, $\rho$ and $V$ the density and velocity, respectively. The subscript $m$ indicates the mixture, and $\rho_{m}$ and $V_{m}$ are given by

$$
\begin{aligned}
& \rho_{m}=\rho_{G} \alpha+\rho_{L}(1-\alpha) \\
& V_{m}=\frac{\rho_{G} \alpha V_{G}+\rho_{L}(1-\alpha) V_{L}}{\rho_{m}}
\end{aligned}
$$

where $\alpha$ is the volume fraction of the gas phase and the subscripts $G$ and $L$ denote the gas and liquid phases, respectively. The mass conservation of the gas phase and the momentum conservation of the mixture are respectively given by

$$
\begin{aligned}
& \frac{\partial}{\partial t}\left(\rho_{G} \alpha\right)+\frac{\partial}{\partial z}\left(\rho_{G} \alpha V_{m}\right)=-\frac{\partial}{\partial z}\left(\frac{\rho_{G} \rho_{L}}{\rho_{m}} \alpha V_{G J}\right) \\
& \frac{\partial}{\partial t}\left(\rho_{m} V_{m}\right)+\frac{\partial}{\partial z}\left(\rho_{m} V_{m}^{2}\right)=-\frac{\partial P}{\partial z}-\frac{4 \tau}{D}-\rho_{m} g-\frac{\partial}{\partial z}\left(\frac{\rho_{G} \rho_{L}}{\rho_{m}} \frac{\alpha}{1-\alpha} V_{G J}^{2}\right)
\end{aligned}
$$


where $\tau$ is the wall shear stress and $V_{G J}$ the averaged drift velocity defined by

$$
V_{G J}=(1-\alpha)\left(V_{G}-V_{L}\right)
$$

which was calculated using the drift-flux model (Zuber and Findlay, 1965):

$$
V_{G J}=\left(C_{0}-1\right)\left(J_{G}+J_{L}\right)+v_{G J}
$$

where $J$ is the volume flux, $C_{0}$ the distribution parameter and $v_{G J}$ the drift velocity. The $C_{0}$ and $v_{G J}$ were given by (Ishii, 1977)

$$
\begin{aligned}
& C_{0}= \begin{cases}1.2-0.2 \sqrt{\rho_{G} / \rho_{L}} & \text { for bubblyflow } \\
1.2 & \text { for slug flow } \\
1.0 & \text { for annularflow }\end{cases}
\end{aligned}
$$

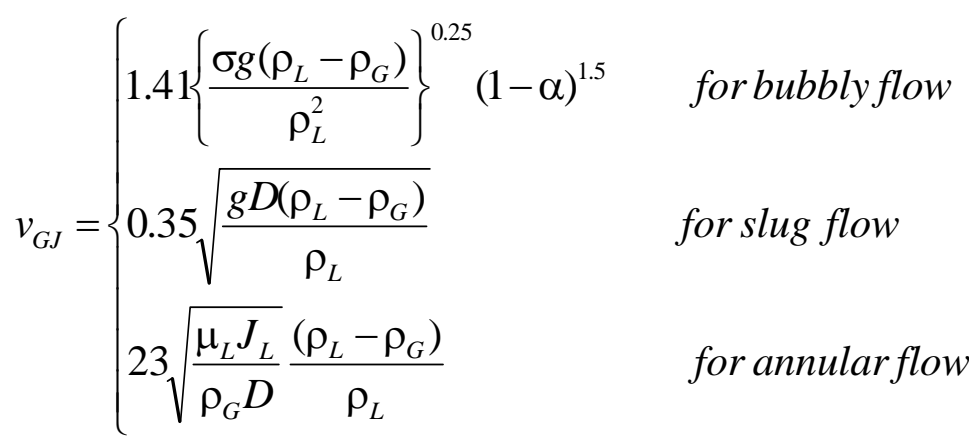

where $\sigma$ is the surface tension and $\mu$ the viscosity. The flow pattern was determined by using the Weisman's correlation (1981, bubbly-slug) and the Wallis correlations (1969, slug-annular). A correlation set for slug/froth flow proposed by Kawanishi et al. (1990) was also used for 
comparison. Their correlation set covers wide flow conditions including high pressure conditions and large diameter tubes. For upward flows, $C_{0}$ is given by

$$
C_{0}=1.2-0.2 \sqrt{\rho_{G} / \rho_{L}}
$$

The $v_{G J}$ depends on the total volume flux $J_{T}\left(=J_{G}+J_{L}\right)$, the pressure $P$ and the pipe diameter $D$ and is summarized in Table 1.

The frictional pressure gradient $4 \tau / D$ in Eq. (5) is given by

$$
\frac{4 \tau}{D}=\frac{\lambda}{D} \frac{\rho_{L} J_{L}^{2}}{2} \Phi_{L}^{2}
$$

where $\lambda$ is the friction factor for single phase flow, $\Phi_{\mathrm{L}}^{2}$ is the two-phase multiplier and is given by the following equation (Akagawa, 1957; Katsuhara, 1958):

$$
\Phi_{L}^{2}=(1-\alpha)^{-1.75}
$$

The Lockhart \& Martinelli method (LM method) for calculating the frictional pressure gradient (Lockhart and Martinelli, 1949) was also used for comparison. The Chisolm’s equation (Chisolm, 1967) was used for $\Phi_{\mathrm{L}}^{2}$.

The SIMPLE algorism (Patankar, 1981) with a staggered grid was adopted for solving the equations. The number of cells was 400 , which was determined from a preliminary sensitivity test for the grid number. The temperature was assumed to be constant and the exit pressure was fixed at the atmospheric pressure. The length and diameter of the pipe, $L(1 \mathrm{~m})$ and $D(8,11$ and $16 \mathrm{~mm})$, the entrance length $L^{\prime}(20 \mathrm{~mm})$, the height $H_{d}(0.3 \mathrm{~m})$ of the exit from the water surface and the 
depth of the gas injection point $H_{s}(0.7 \mathrm{~m})$ were set at the same values as the experiments. The gas density $\rho_{G}$ was calculated using the equation of state for an ideal gas, while the liquid density $\rho_{L}$ was fixed at a constant value.

Results of the numerical predictions at $T_{0}=298$ and $313 \mathrm{~K}$ are shown in Fig. 7 together with the experiments. The difference between the predictions based on Hatakeyama's method at $T_{0}=298$ at $313 \mathrm{~K}$ is very small. This is the same as the experimental results, and indicates that the changes in fluid properties does not affect $Q_{L}$ in the present experimental range. The predictions reasonably agree with the experimental results for all the conditions. The predictions with the Lockhart \& Martinelli method tend to overestimate $Q_{L}$, in other words, underestimate $\Phi_{L}^{2}$ except for $D=16$ $\mathrm{mm}$. There is no difference between the prediction with the Hatakeyama's correlation set and that with the Kawanishi's correlation set except for a low $Q_{g}$ region of $D=16 \mathrm{~mm}$. This is because both correlation sets adopt the same equation for the slug flow regime. On the other hand, the Kawanishi’s correlation set uses interporation of equations for bubbly and slug flows in Eq. (8) in the low $Q_{g}$ region for $D=16 \mathrm{~mm}$. Since the correlation set covers wide ranges of $D$ and pressure, it would be applicable to large-scale systems too.

Figure 8 shows a comparison between the predictions and experimental results (Sato et al., 1986) for a large scale air-lift pump. The diameter and the length of the main pipe are $151.1 \mathrm{~mm}$ and 196.6 m, respectively. Although all predictions reasonably agree with the experimental result, the predictions with the Lockhart \& Martinelli method tend to underestimate $Q_{L}$. The Kawanishi’s correlation set gives good predictions for a high $Q_{G}$ range and it tends to underestimate $Q_{L}$ in low $Q_{G}$ conditions. The prediction with the Hatakeyama's correlation set gives the best result among the three methods. Hence, the one-dimensional drift flux model with the Hatakeyama's correlation set can be used to evaluate performance of the gaslift pump making use of phase change, whereas flows in the other parts and conservation of thermal energy have to be considered to evaluate the performance of the system. 
Since the performance of the present gaslift pomp is confirmed to be the same as that of an airlift pump using compressed air with the same geometry of the main pipe at the same $J_{G}$, the ratio of efficiencies of these two pumps is the same as the inverse ratio of energies required for supplying gas to the main pipe. For the standard airlift pump, the energy is mostly used for compressing air. The energy $E_{C A, a d}$ required for adiabatically compressing atmospheric air (pressure: $P_{0}$, temperature: $T_{0}$ ) to the pressure $P_{I}$ at the air injection point of the main pipe is

$$
E_{C A, a d}=W_{A} \frac{\kappa}{\kappa-1} \frac{P_{0}}{\rho_{A 0}}\left\{\left(\frac{P_{I}}{P_{0}}\right)^{\frac{\kappa-1}{\kappa}}-1\right\}
$$

where $W_{A}$ is the mass flow rate of air, $\rho_{A 0}$ the density of air at the atmospheric pressure and $\kappa$ the ratio of specific heats. The energy ECA supplied to a compressor can be evaluated by

$$
E_{C A}=\frac{E_{C A, a d}}{\eta_{C A}}=\frac{W_{A}}{\eta_{C A}} \frac{\kappa}{\kappa-1} \frac{P_{0}}{\rho_{A, 0}}\left\{\left(\frac{P_{I}}{P_{0}}\right)^{\frac{\kappa-1}{\kappa}}-1\right\}
$$

where $\eta_{C A}$ is the adiabatic efficiency of the compressor. On the other hand, most of the energy is consumed for condensing the working fluid in the proposed gaslift pump. The energy $E_{C F, \text { cond }}$ required for the condensation can be evaluated by

$$
E_{C F, \text { cond }}=W_{W F}\left\{C_{p G}\left(T_{I N}-T_{B, C}\right)+L+C_{L}\left(T_{B, C}-T_{O U T}\right)\right\}
$$

where $W_{W F}$ is the mass flow rate of the working fluid, $C_{p G}$ the specific heat of the gas phase at constant pressure, $L$ the latent heat, $C_{L}$ the specific heat of the liquid phase, $T_{I N}$ the temperature at the inlet of the condenser, $T_{B, C}$ the boiling (saturation) temperature at the condenser and TouT the 
temperature at the exit of the condenser which is the same as that at the inlet of the evaporator, provided that the thermal insulation of the adiabatic pipe is perfect. The working fluid must be liquid at the exit of the condenser, and therefore, TouT have to be lower than $T_{B, C}$. In the most efficient case, $T_{\text {OUT }}$ is the same as $T_{B, C}$. Since the working fluid contacts with the surrounding liquid in the main pipe, $T_{I N}$ is nearly equal to the temperature $T_{I}$ at the air injection point of the main pipe (or lower than $T_{I}$ due to expansion of the gas) when we use an adiabatic pipe for the main pipe. Hence, Eq. (14) can be rewritten as

$$
E_{C F, \text { cond }}=W_{W F}\left\{C_{p G}\left(T_{I}-T_{B, C}\right)+L\right\}
$$

If the Clapeyron-Clausius equation holds and $L$ is constant in the proposed pump system, the following relation between the pressure and the temperature is obtained:

$$
\ln \frac{P_{I}}{P_{0}}=-\frac{L}{R_{0}}\left\{\frac{1}{T_{I}}-\frac{1}{T_{B, 0}}\right\}
$$

where $R_{0}$ is the gas constant and $T_{B, 0}$ the boiling temperature at the atmospheric pressure. Since the pressure in the condenser is close to the atmospheric pressure, $T_{B, C} \simeq T_{B, 0}$. Thus, substituting Eq. (16) into Eq. (15) yields

$$
E_{C F, \text { cond }}=W_{W F}\left\{C_{p G} \frac{R_{0}}{L} T_{B, 0} T_{I} \ln \frac{P_{I}}{P_{0}}+L\right\}=W_{W F}\left\{C_{p G} \frac{P_{0}}{L \rho_{G, 0}} T_{I} \ln \frac{P_{I}}{P_{0}}+L\right\}
$$

where $\rho_{G, 0}$ is the gas density at the atmospheric pressure. The energy $E_{C F}$ supplied to the condenser can be evaluated by 


$$
E_{C F}=\frac{E_{C F, \text { cond }}}{\eta_{C D}}=\frac{W_{W F}}{\eta_{C D}}\left\{C_{p G} \frac{P_{0}}{L \rho_{G, 0}} T_{I} \ln \frac{P_{I}}{P_{0}}+L\right\}
$$

where $\eta_{C D}$ is the coefficient of performance of the condenser. Hence, the ratio $R_{\eta}$ of the efficiency of the present pump to the standard airlift pump can be evaluated by

$$
R_{\eta}=\frac{E_{C A}}{E_{C F}}=\frac{\eta_{C D}}{\eta_{C A}} \frac{W_{A}}{W_{W F}} \frac{\kappa}{\kappa-1} \frac{\left\{\left(\frac{P_{I}}{P_{0}}\right)^{\frac{\kappa-1}{\kappa}}-1\right\}}{\left\{C_{p G} \frac{\rho_{A, 0}}{\rho_{G, 0}} \frac{T_{I}}{L} \ln \frac{P_{I}}{P_{0}}+\frac{\rho_{A, 0} L}{P_{0}}\right\}}
$$

Since the volume flow rate $Q_{G}\left(=W_{W F} / \rho_{G}\right)$ of the working fluid in the present pump is equal to $Q_{A}$ ( $\left.=W_{A} / \rho_{A}\right)$ in the standard airlift pump, Eq. (19) can be rewritten as

$$
R_{\eta}=\frac{\eta_{C D}}{\eta_{C A}} \frac{\kappa}{\kappa-1} \frac{\left\{\left(\frac{P_{I}}{P_{0}}\right)^{\frac{\kappa-1}{\kappa}}-1\right\}}{\left\{C_{p G} \frac{T_{I}}{L} \ln \frac{P_{I}}{P_{0}}+\frac{\rho_{G 0} L}{P_{0}}\right\}}
$$

Figure 9 shows $R_{\eta}$ for gaslift pumps using HFC-245fa and Xenon as the working fluid, physical properties of which are shown in Table 2. $\eta_{C A}, \eta_{C D}$ and $T_{I}$ are assumed to be 0.8, 1.2 and $277 \mathrm{~K}$, respectively. $R_{\eta}$ increases with $P_{I} / P_{0}$, i.e. with the depth of water. Although $R_{\eta}$ is lower than unity

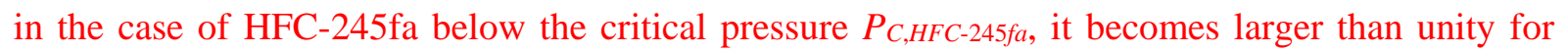
$P_{I} / P_{0}>17$ in the case of Xenon. This indicates that the proposed gaslift pump has an efficiency higher than a standard airlift pump when applying to a deep sea area, provided that an appropriate working fluid covering the requirements listed in the section 2 is selected. The boiling point at the 
evaporator have to be lower than the surrounding water temperature. The boiling point of Xenon is $170 \mathrm{~K}$ at the atmospheric pressure and it becomes about $275 \mathrm{~K}$ at $P_{\mathrm{I}} / P_{0} \sim 45\left(H_{\mathrm{S}} \sim 450 \mathrm{~m}, T_{I} \sim 277\right.$ K). Hence, Xenon can be boiled even in the deep sea area, whereas it should be cooled to $170 \mathrm{~K}$ at the condenser (Eq. (20) takes this into account). Selection of appropriate working fluid is required if the depth of water, i.e., pressure and temperature, at the evaporator is changed. Critical point of working fluid restricts the applicable depth range of the present gaslift pump and the standard airlift pump as well. The critical point of Xenon $P_{C, X e n o n}$ is higher than that of air $P_{C, A i r}$, and therefore, the gaslift pump using Xenon is applicable to sea depth deeper than the standard airlift pump. Although further researches are indispensable to develop a large scale system based on the present gaslift pump and to evaluate its performance, the above discussion indicates the potential of the efficient gaslift pump by making use of the phase change of the working fluid.

\section{Conclusion}

We proposed a gaslift pump utilizing phase change of working fluid to avoid large work for compressing air. A small-scale gaslift pump using a hydro-fluorocarbon as the working fluid was developed to examine the feasibility of the proposed system. Four Peltier devices were used to cool the hydro-fluorocarbon gas. The flow rates of the drawn water were measured at various flow rates of the working fluid using pipes with three different diameters. The experiments using injected air were also carried out for comparison. As a result, the feasibility of the pump was confirmed and the relation between flow rates of the drawn water and working fluid was the same as the airlift pump driven by compressed-air injection. Numerical simulations using a method proposed by Hatakeyama were also carried out. The method gave good predictions for the relation between the flow rates of drawn water and working fluid in the present system. The discussion on the efficiency showed that the proposed gaslift pump has an efficiency higher than a standard airlift pump when applying to a deep sea area, provided that an appropriate working fluid is selected. 


\section{Acknowledgements}

This work was supported by Japan Science and Technology Agency (Adaptable and Seamless Technology Transfer Program through Target-driven R\&D, AS262Z00473K).

\section{References}

Akagawa, K., 1957. The Flow of the Mixture of Air and Water: III. The Friction Losses in Horizontal, Inclined and Vertical Tubes. Trans. JSME. 23 (128), 292 - 298.

Chisholm, D., 1967, A Theoretical Basis for the Lockhart-Martinelli Correlation for Two-Phase Flow, International Journal of Heat and Mass Transfer, 10, 1767- 1778.

Cho, N.C., Hwang, I,J., Lee, C.M., Park, J.W., 2009, An Experimental Study on the Airlift Pump with Air Jet Nozzle and Booster Pump. J. Environmental Sciences, Supplement, S19 - S23.

Hatakeyama, N., Masuyama, T., 1989. Numerical Simulation for Lifting Characteristics of Water with Large Scale Air-Lift Pump. Shigen-to-Sozai. 105, 14, 1051 - 1057.

Hatakeyama, N., Takahashi, H., Saito, T., Masuyama, T., 1999, A Numerical Simulation of Unsteady Flow in an Air-lift Pump for Lifting Deep-sea Mineral Resources, Shigen-to-Sozai, $115,958-964$.

Ishii, M., 1977. One-dimensional Drift-flux Model and Constitutive Equations for Relative Motion between Phases in Various Two-phase Flow Regimes. ANL-77-47.

Kassab, S., Kaandill, H.A., Warda, H.A., Ahmed, W.H., 2007, Experimental and Analytical Investigations of Airlift Pumps Operating in Three-Phase Flow. Chem. Eng. J., 131, 273 - 281.

Kassab, S.Z., Kandil, H.A., Warda, H.A, Ahmed, W.H., 2009, Air-Lift Pumps Characteristics under Two-Phase Flow Conditions. Int. J. Heat and Fluid Flow. 30, 88 - 98.

Katsuhara, T., 1958. Influences of Roughness of Inner Surface of Pipe upon Pressure Drop due to Friction in Two-Phase Flow. Trams. JSME. 24 (148), 1050 - 105. 
Kawanishi, K., Hirao, Y., Tsuge, A., 1990. An experimental study on Drift Flux parameters for Two-Phase flow in vertical round tubes. Nuclear Engineering and Design. 120, 447-458.

Lockhart, R. W., Martinelli, R. C., 1949. Proposed Correlation of Data for Isothermal Two Phase Flow. Chemical Engineering Progress, 45, 39-48.

Patankar, S.V., 1981, A Calculation Procedure for Two-Dimensional Elliptic Situations, Numerical Heat Transfer, 4, 409-425.

Rona, P.A., 2003, Resources of the sea floor. Science, 299, 673 - 674.

Saito, T., 1986. Manganese Nodules Mining System -Status and Some Results of R \& D-. Journal of the Japan Society of Mechanical Engineers, 89, 1040 - 1045.

Saito, T., Tomishima, Y., Yamazaki, T., Usami, T., Yokokawa, A., Shimizu, Y., 1986, Saiko-toHoan, 32, 10, $36-47$.

Sadatomi, M., Kawahara, A., Kumura, T., Nakao, J., 2008, Development of a Bubble-jet-type Airlift-pump (Feasibility Test and Performance Prediction), Transactions of JSME, 74, 1263 1269.

Sadatomi, M., Kawahara, A., Nishiyama, T., 2013, Bubble-Jet-Type Air-Lift Pump for Pumping Water and Sediments Deposited on Lake and Sea Beads, Japanese Journal of Multiphase Flow, 27, 2, $141-151$.

Todoroki, I, Sato, Y., Honda, T., 1972. Pumping Characteristics of Airlift Pump. Transactions of JSME. 38, 2085 - 2092.

Tomiyama, A., Minagawa, H., Sakaguchi, T., 1991, Derivation of Constitutive Equations for Interfacial Momentum Transfer Required for the Analyses of Gas-liquid-Solid Three-Phase Flow with a One-Dimensional Three-Fluid Model, Trans. JSME, 57, 536, 1239 - 1245.

Weisman, J., Kang, S.A., 1981. Flow Patten Transitions in Vertical and Upwardly Inclined Pipes, International Journal of Multiphase Flow, 7, 271 - 291.

Wallis, G.B., 1969. One-Dimensional Two-Phase Flow. Academic press. 
Zuber, N., Findlay, J.A., 1965. Average Volumetric Concentration in Two-Phase Flow Systems, Journal of Heat Transfer, 87, 453-468. 
Figure caption

Table 1 Drift velocity correlations proposed by Kawanishi et al. (1990)

Table 2 Physical properties of working fluids

Fig. 1. Concept of gaslift pump.

Fig. 2. Experimental apparatus.

Fig. 3 Examples of time variations of temperatures and flow rate $(D=11 \mathrm{~mm})$.
(a) Temperatures
(b) Flow rate

Fig. 4 Flow patterns.
(a) $D=6 \mathrm{~mm}$ (b) $D=11 \mathrm{~mm}$
(c) $D=16 \mathrm{~mm}$

Fig. 5 Flow rates of drawn water.

Fig. 6 Simulated domain.

Fig. 7 Comparison between predictions and experiments.

Fig. $8 Q_{L}$ in large scale experiment by Saito et al. (1986).

Fig. 9 Efficiency ratio of the present gaslift pump to a standard airlift pump. 
Table 1 Drift velocity correlations proposed by Kawanishi et al. (1990)

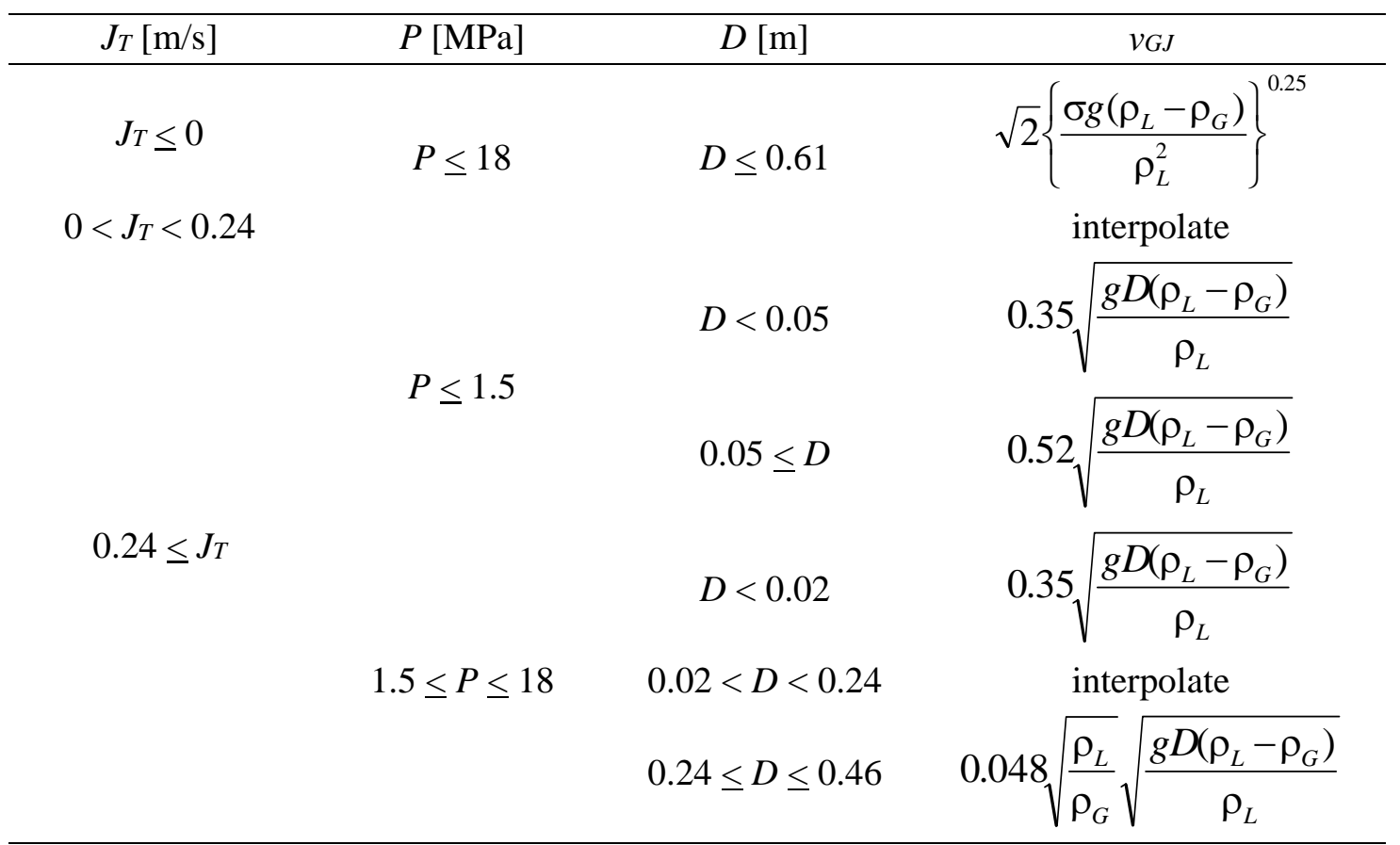


Table 2 Physical properties of working fluids

\begin{tabular}{l|cccc}
\hline & $C_{p G}[\mathrm{~kJ} / \mathrm{kg} / \mathrm{K}]$ & $\rho_{G, 0}\left[\mathrm{~kg} / \mathrm{m}^{3}\right]$ & $L[\mathrm{~kJ} / \mathrm{kg}]$ & $P_{C}[\mathrm{MPa}]$ \\
\hline \hline Air & 1.01 & 1.18 & - & 3.77 \\
HFC-245fa & 0.88 & 5.92 & 196.7 & 3.64 \\
Xenon & 0.16 & 5.39 & 99.2 & 5.84 \\
\hline
\end{tabular}




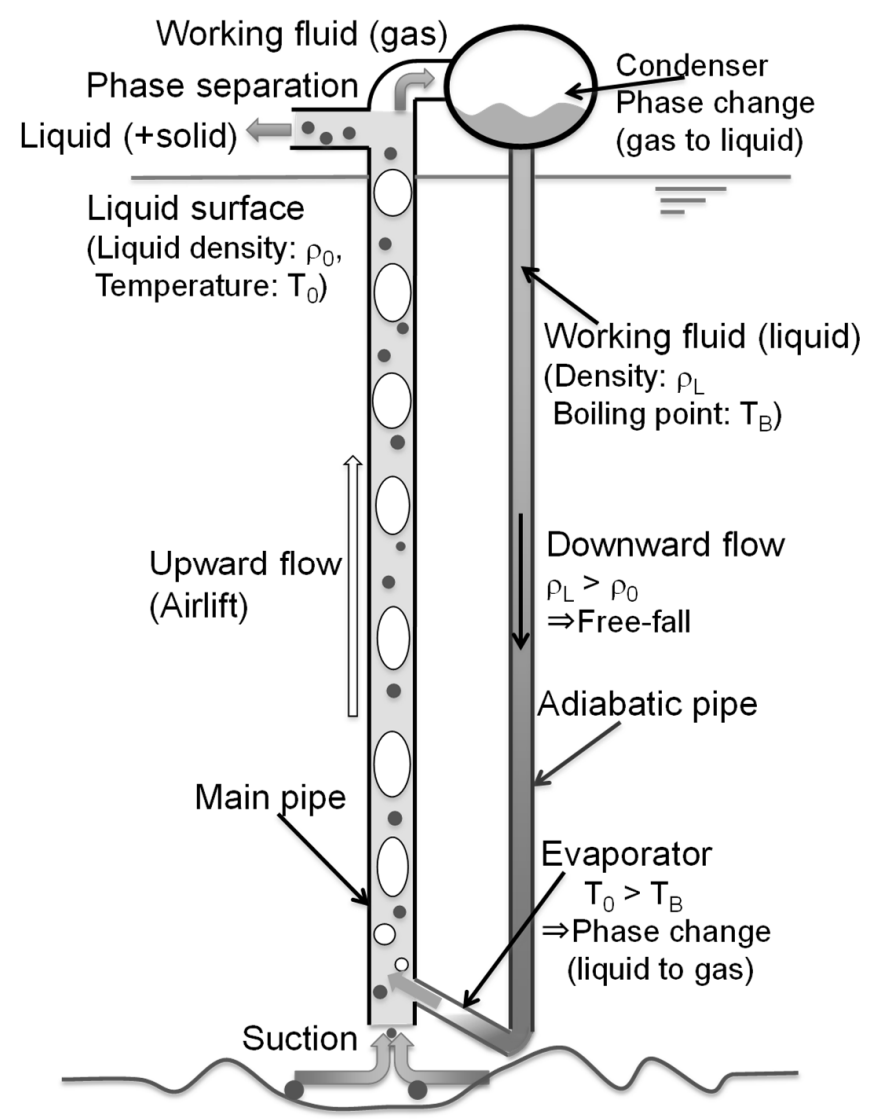

Fig. 1. Concept of gaslift pump. 


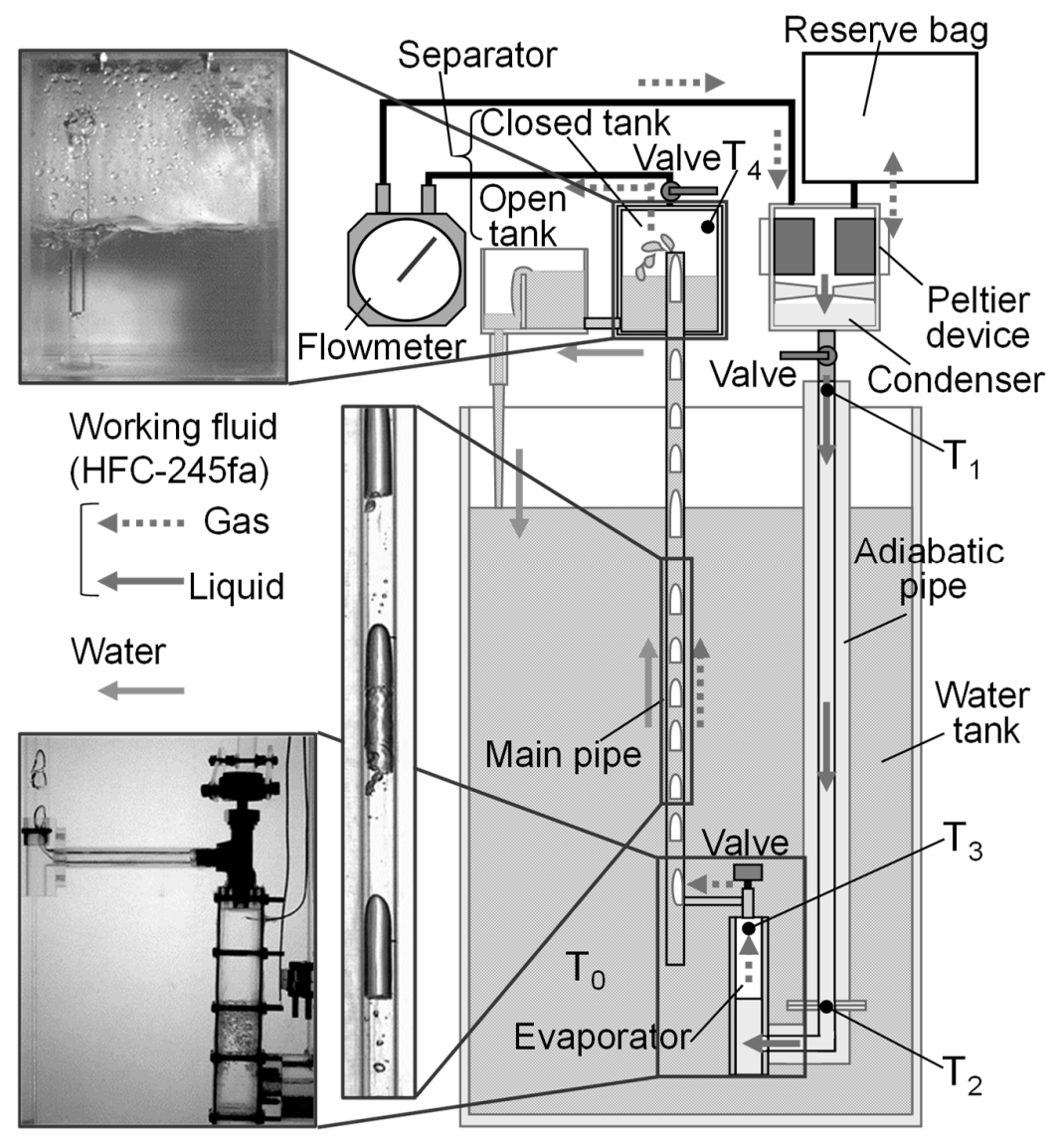

Fig. 2. Experimental apparatus. 


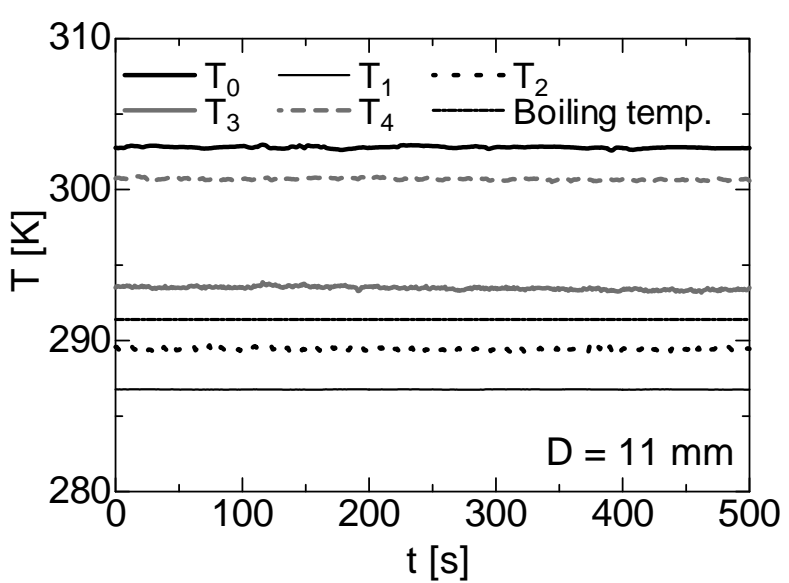

(a) Temperatures

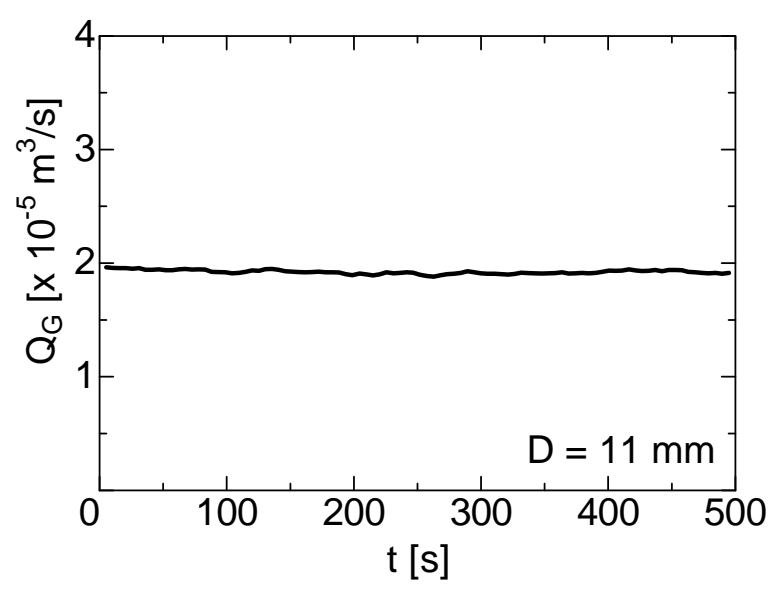

(b) Flow rate

Fig. 3 Examples of time variations of temperatures and flow rate $(D=11 \mathrm{~mm})$. 


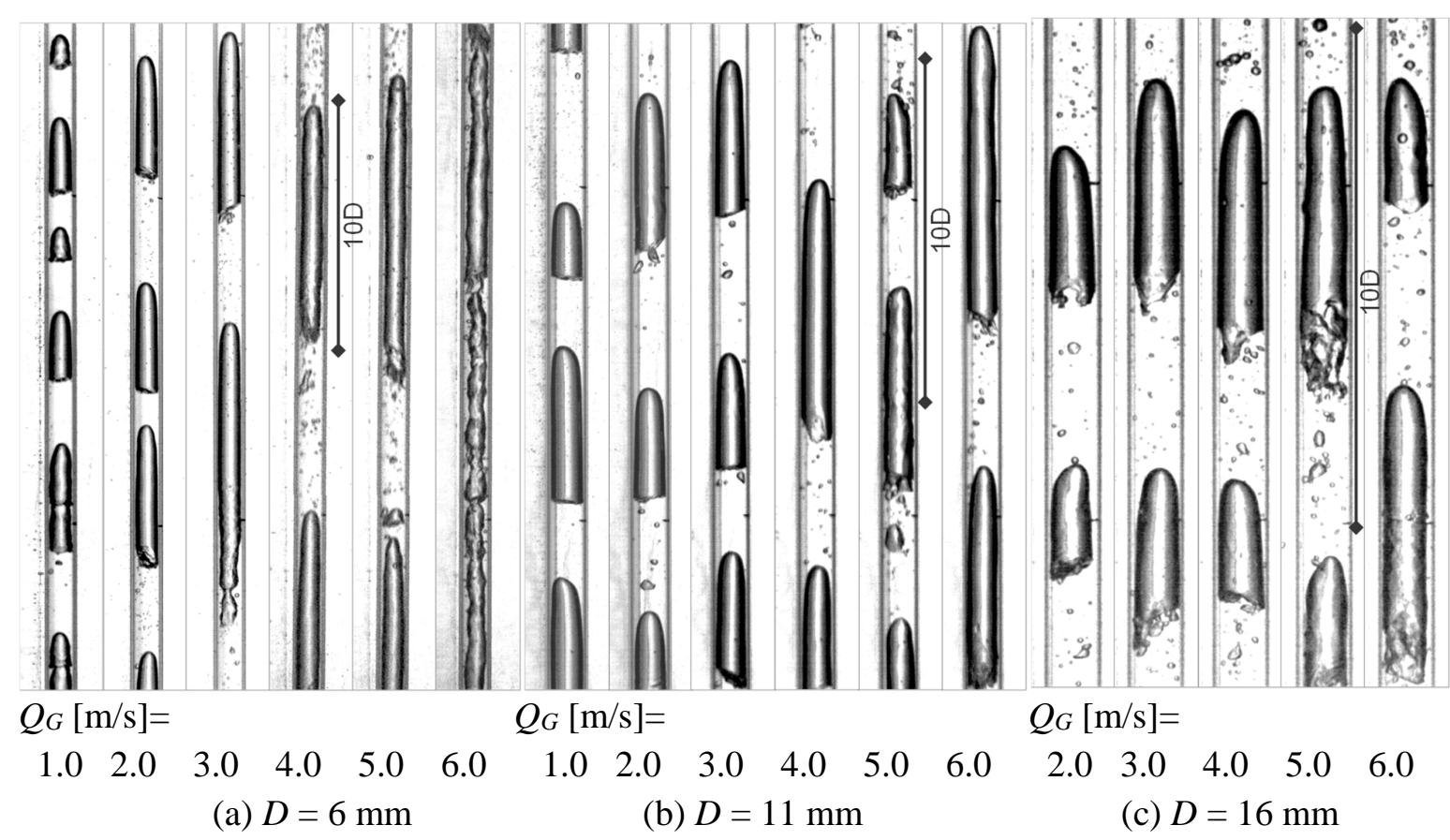

Fig. 4 Flow patterns. 


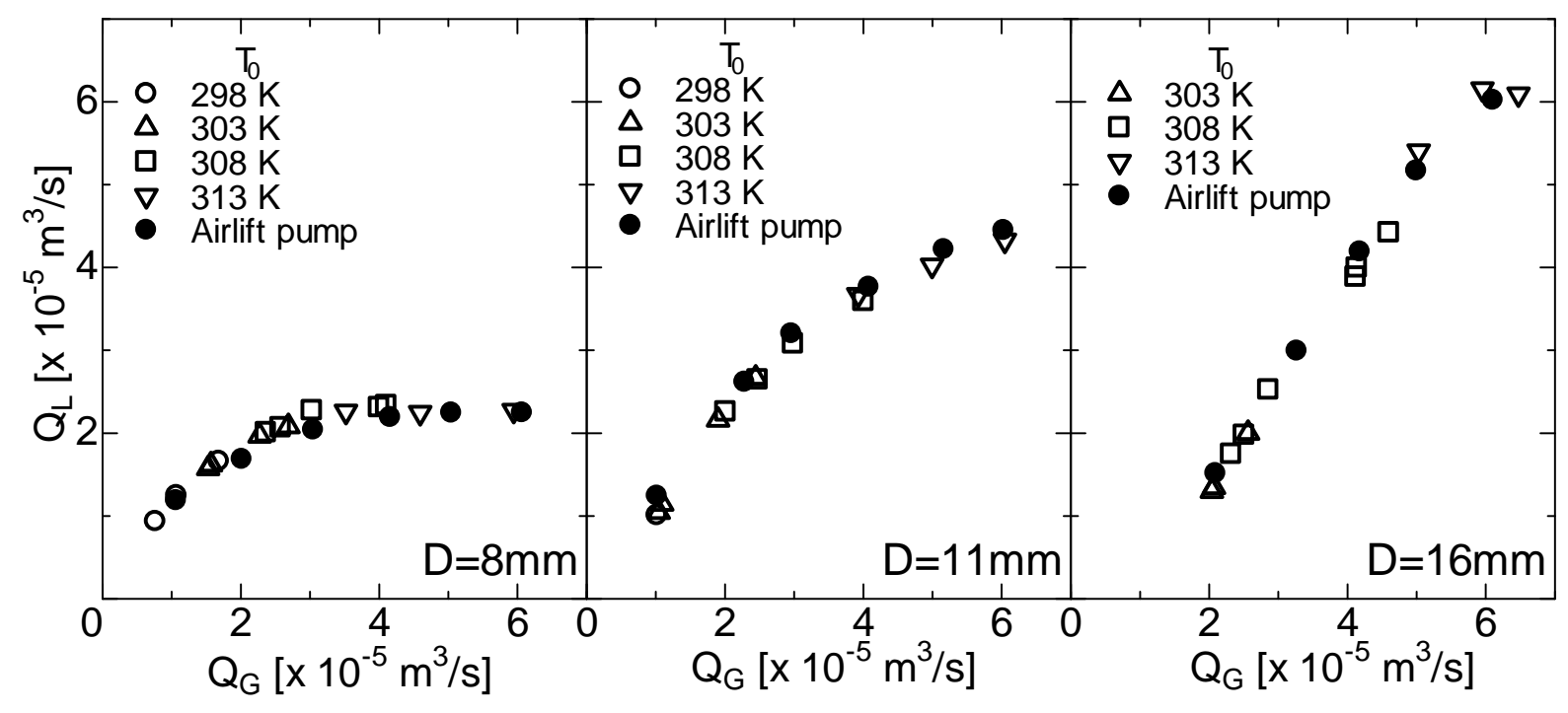

Fig. 5 Flow rates of drawn water. 


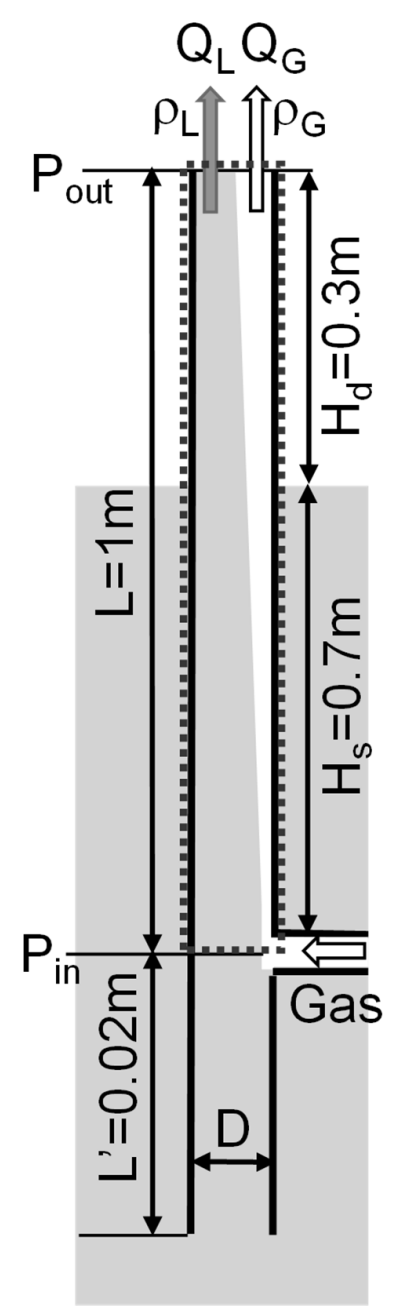

Fig. 6 Simulated domain. 


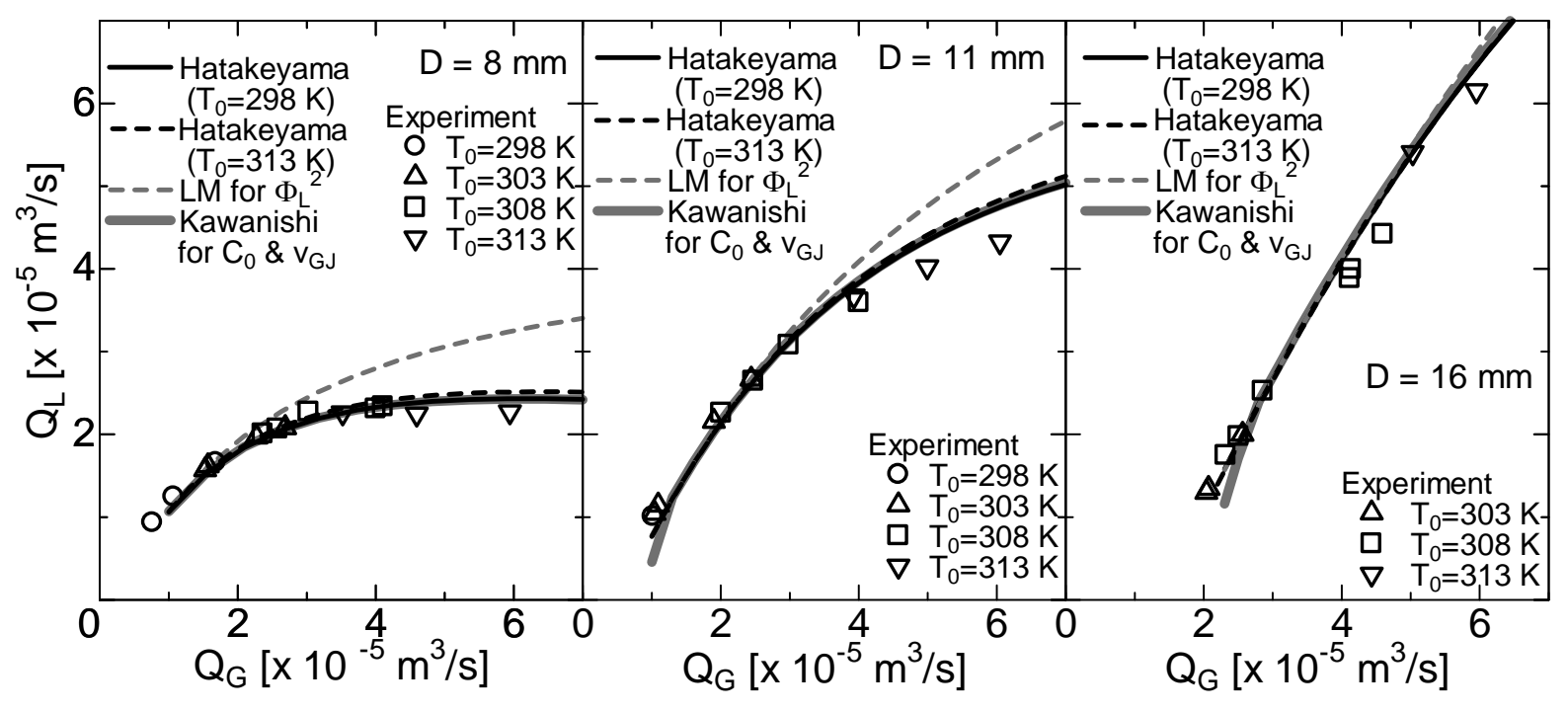

Fig. 7 Comparison between predictions and experiments. 


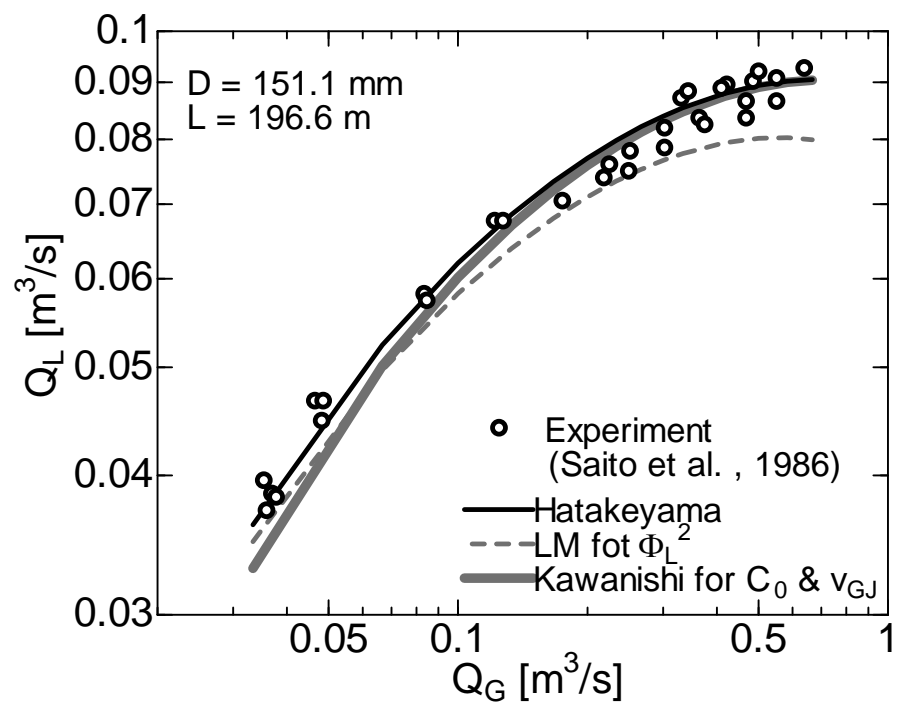

Fig. $8 Q_{L}$ in large scale experiment by Saito et al. (1986). 


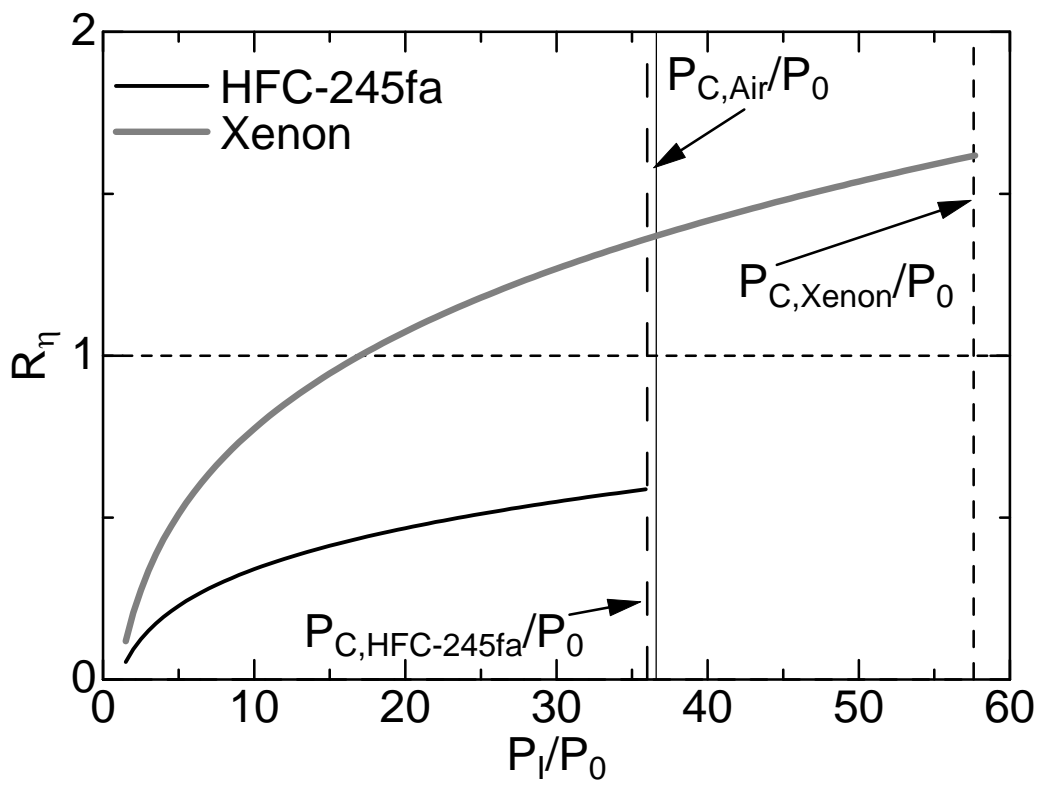

Fig. 9 Efficiency ratio of the present gaslift pump to a standard airlift pump. 